\title{
Watch out for slowly progressive weakness of the distal upper limb: it could be chronic acquired demyelinating neuropathy!
}

\author{
So Young Kwak ${ }^{1}$, Mathieu Boudier-Revéret ${ }^{2}$, Min Cheol Chang ${ }^{1}$ \\ ${ }^{1}$ Department of Physical Medicine and Rehabilitation, College of Medicine, Yeungnam University, Taegu, Republic of Korea; ${ }^{2}$ Department of \\ Physical Medicine and Rehabilitation, Centre hospitalier de l'Université de Montréal, Montreal, Canada \\ Correspondence to: Min Cheol Chang, MD. Department of Physical Medicine and Rehabilitation, College of Medicine, Yeungnam University 317-1, \\ Daemyungdong, Namku, Taegu, 705-717, Republic of Korea. Email: wheel633@ynu.ac.kr.
}

Submitted Feb 07, 2020. Accepted for publication Mar 04, 2020.

doi: 10.21037/apm.2020.04.15

View this article at: http://dx.doi.org/10.21037/apm.2020.04.15

In clinical practice, physicians occasionally encounter patients with complaints of progressive weakness of the hand or wrist. Distal upper limb weakness is commonly caused by compressive neuropathy of the spinal cord (i.e., cervical spondylotic myelopathy), spinal nerve roots (i.e., foraminal stenosis), or peripheral nerves (i.e., tardy ulnar neuropathy, carpal tunnel syndrome, thoracic outlet syndrome, or anterior/posterior interosseous syndromes). However, forms of chronic acquired demyelinating neuropathy, such as multifocal motor neuropathy (MMN) and multifocal acquired demyelinating sensory and motor neuropathy (MADSAM), are occasionally the cause of distal upper limb weakness. However, many clinicians lack sufficient knowledge about the diagnosis of MMN and MADSAM. Consequently, patients with motor weakness caused by these disorders are frequently misdiagnosed and go without treatment for extended periods of time. Here, we present a case each of MMN and MADSAM; in both cases, the condition was diagnosed several years after symptom presentation.

A 56-year-old man (patient 1) visited the physical medicine and rehabilitation department of our hospital, presenting with weakness in both hands and wrists that had progressed slowly over the previous 7 years. We evaluated the weakness in the bilateral fingers, bilateral wrist flexors, and right finger extensors. Weakness was found to be asymmetric between the right and left upper limbs. The strength scores on the medical research council (MRC) scale were $3 / 5$ and $4 / 5$ for the right and left sides, respectively. Mild atrophy was noted in the hand muscles, bilaterally. No sensory deficit was present, nor were Tinel's or Hoffman's signs. The muscle stretch reflexes of the biceps and triceps were normal. Electrophysiological examination (Table 1) showed decreases in the conduction velocity and amplitude of the compound motor action potential (CMAP) for the bilateral median and right ulnar nerves. It also showed a conduction block in the bilateral median nerves. Sensory nerve action potentials (SNAPs) in both upper limbs showed no abnormalities. Electromyography (EMG) revealed positive sharp waves $(2+)$ on the right side, in the flexor carpi radialis, flexor carpi ulnaris, abductor pollicis brevis, first dorsal interossei, and abductor digiti minimi. On the left side, it revealed positive sharp waves (1+) only in the abductor pollicis brevis. Cervical magnetic resonance imaging (MRI) showed no abnormalities. Anti-GD1b IgM, anti-GM1 IgG, and anti-GM1 IgM antibodies were not detected. Cerebrospinal fluid examination revealed no abnormalities. MMN was diagnosed using established diagnostic criteria (1). We administered $140 \mathrm{~g}(2 \mathrm{~g} / \mathrm{kg})$ intravenous immunoglobulin (IVIg) over 5 days. At a follow-up two weeks after treatment completion, it was observed that the patient had almost completely recovered from motor weakness. This treatment was followed by monthly infusions of $70 \mathrm{~g}(1 \mathrm{~g} / \mathrm{kg}) \mathrm{Ig}$. At the 6-month follow-up, his recovered motor function was maintained.

Another 56-year-old man (patient 2) visited the physical medicine and rehabilitation department of our hospital, presenting with right hand and wrist weakness that had progressed slowly over the previous 10 years. MRC strength scores were $3 / 5$ for each of the right finger extensor, wrist extensor, and wrist flexor. Hypoesthesia and hypoalgesia were present nearly throughout the entire right hand. 
Table 1 Results of the nerve conduction study

\begin{tabular}{|c|c|}
\hline Nerve (normal values) & Results \\
\hline \multicolumn{2}{|l|}{ Patient 1 (multifocal motor neuropathy) } \\
\hline \multicolumn{2}{|l|}{ Compound motor action potential } \\
\hline \multicolumn{2}{|l|}{ Rt. median } \\
\hline CMAP amp D/E (>5.0), mV & 10.9/1.9 \\
\hline Percentage of conduction block E-D & 83 \\
\hline MNCV (>50), ms & 40 \\
\hline \multicolumn{2}{|l|}{ Rt. ulnar } \\
\hline CMAP amp D/E (>5.0), mV & $7.0 / 5.8$ \\
\hline Percentage of conduction block E-D & - \\
\hline MNCV $(>50), \mathrm{ms}$ & 46 \\
\hline \multicolumn{2}{|l|}{ Lt. median } \\
\hline CMAP amp D/E (>5.0), mV & $10.4 / 3.2$ \\
\hline Percentage of conduction block E-D & 69 \\
\hline $\operatorname{MNCV}(>50), \mathrm{ms}$ & 40 \\
\hline \multicolumn{2}{|l|}{ Lt. ulnar } \\
\hline CMAP amp D/E (>5.0), mV & $18.5 / 17.6$ \\
\hline Percentage of conduction block E-D & - \\
\hline $\operatorname{MNCV}(>50), \mathrm{ms}$ & 59 \\
\hline \multicolumn{2}{|l|}{$\begin{array}{l}\text { Patient } 2 \text { (multifocal acquired demyelinating } \\
\text { sensory and motor neuropathy) }\end{array}$} \\
\hline \multicolumn{2}{|l|}{ Compound motor action potential } \\
\hline \multicolumn{2}{|l|}{ Rt. median } \\
\hline MNDL (<4.2), ms & 5.5 \\
\hline CMAP amp D/E (>5.0), mV & $2.1 / 1.5$ \\
\hline Percentage of conduction block E-D & - \\
\hline $\operatorname{MNCV}(>50), \mathrm{ms}$ & 25 \\
\hline \multicolumn{2}{|l|}{ Rt. radial } \\
\hline $\operatorname{MNDL}(<4.2), \mathrm{ms}$ & 2.9 \\
\hline CMAP amp D/E (>5.0), mV & $4.2 / 1.7$ \\
\hline Percentage of conduction block E-D & 60 \\
\hline $\operatorname{MNCV}(>50), \mathrm{ms}$ & 51 \\
\hline \multicolumn{2}{|l|}{ Rt. ulnar } \\
\hline MNDL (<4.2), ms & 4.4 \\
\hline CMAP amp D/E (>5.0), mV & $0.7 / 0.3$ \\
\hline Percentage of conduction block E-D & 57 \\
\hline
\end{tabular}

Table 1 (continued)
Table 1 (continued)

\begin{tabular}{cc}
\hline Nerve (normal values) & Results \\
\hline MNCV $(>50), \mathrm{ms}$ & 36 \\
Sensory nerve action potential &
\end{tabular}

No response

Rt. median

Rt. supf. radial

SNAP amp (>20), $\mu \mathrm{V}$

5

Rt. ulnar

No response

CMAP, compound motor action potential; amp, amplitude; D, distal; E, elbow; E-D, elbow to distal segment; MNCV, motor nerve conduction velocity; MNDL, motor nerve distal latency; SNAP, sensory nerve action potential.

Mild global atrophy was also noted in the right hand. Tinel's and Hoffman's signs were not present. The muscle stretch reflexes of the biceps and triceps were normal. Electrophysiological examination (Table 1) showed decreases in the amplitude of the CMAP for the right median, ulnar, and radial nerves. Increased distal latency and decreased conduction velocity were observed in the right median and ulnar nerves. Furthermore, conduction blocks were noted in the right ulnar and radial nerves. SNAPs were not detected in the right median and ulnar nerves and the amplitude in the superficial radial nerve was reduced. EMG revealed positive sharp waves $(3+)$ in the right flexor carpi radialis, flexor carpi ulnaris, extensor carpi radialis longus, abductor pollicis brevis, first dorsal interossei, and abductor digiti minimi. Nerve conduction examinations and an EMG of the left upper limb showed no abnormalities. Cervical MRI showed no abnormalities. We diagnosed the patient with MADSAM based on established diagnostic criteria (2,3). We began administering $100 \mathrm{mg}$ /day oral prednisolone each morning in a single dose. At the 2-week follow-up after the initiation of oral prednisolone, the patient's motor function had almost returned to normal and the dose was decreased to $100 \mathrm{mg}$ every other day. One month after treatment began, we began tapering the dose by $5 \mathrm{mg}$ every 2 weeks. During tapering, the patient's motor weakness did not return. At the 1-month follow-up, his recovered motor function was maintained.

MMN and MADSAM are representative forms of chronic acquired demyelinating neuropathy. They are characterized by asymmetric weakness and are typically distributed in individual peripheral nerves $(4,5)$. While MMN afflicts motor nerves without involving sensory 
nerves, MADSAM results in sensory nerves lesions $(4,5)$. The age of onset varies widely between these conditions. However, it is known that, on average, MMN appears in the early 40s, whereas MADSAM typically appears in the early 50 s $(4,5)$. In both disorders, the distal upper limb of one side is affected, followed by weakness progressing to the other limbs $(4,5)$. Less frequently, these conditions can begin in a distal lower limb. Therefore, MMN and MADSAM can be said to present initially as hand or wrist weakness, and occasionally, as foot weakness. Because these disorders are rare compared to compressive neuropathy, they are infrequently among clinicians' initial potential diagnoses for new patients with distal limb weakness. However, if the progression of motor weakness is slow and it affects more than one individual peripheral nerve, it should alert the clinician to the possibility of MMN or MADSAM. Many clinicians do not have adequate knowledge about these disorders and diagnosis is often delayed as a result. On average, a correct diagnosis of either condition is made 7-10 years after symptom onset. Because MMN and MADSAM usually show excellent treatment outcomes in response to IVIg and corticosteroid treatment, respectively, it is important that clinicians familiarize themselves with the presentation of these disorders.

\section{Acknowledgments}

Funding: The present study was supported by a National Research Foundation of Korea grant funded by the Korean government (grant no. NRF-2019R1F1A1061348).

\section{Footnote}

Conflicts of Interest: All authors have completed the ICMJE uniform disclosure form (available at http://dx.doi. org/10.21037/apm.2020.04.15). The authors have no conflicts of interest to declare.

Cite this article as: Kwak SY, Boudier-Revéret M, Chang MC. Watch out for slowly progressive weakness of the distal upper limb: it could be chronic acquired demyelinating neuropathy! Ann Palliat Med 2020;9(3):1285-1287. doi: 10.21037/ apm.2020.04.15
Ethical Statement: The authors are accountable for all aspects of the work in ensuring that questions related to the accuracy or integrity of any part of the work are appropriately investigated and resolved.

Open Access Statement: This is an Open Access article distributed in accordance with the Creative Commons Attribution-NonCommercial-NoDerivs 4.0 International License (CC BY-NC-ND 4.0), which permits the noncommercial replication and distribution of the article with the strict proviso that no changes or edits are made and the original work is properly cited (including links to both the formal publication through the relevant DOI and the license). See: https://creativecommons.org/licenses/by-nc-nd/4.0/.

\section{References}

1. Olney RK, Lewis RA, Putnam TD, et al. American Association of Electrodiagnostic Medicine Consensus criteria for the diagnosis of multifocal motor neuropathy. Muscle Nerve 2003;27:117-21.

2. Amato AA, Dumitru D. Acquired neuropathies.In: Dumitru D, Amato AA, Zwarts M. editors. Electrodiagnostic Medicine, 2nd ed. Philadelphia: Hanley \& Belfus, 2001;959.

3. Joint Task Force of the EFNS and the PNS.. European Federation of Neurological Societies/Peripheral Nerve Society Guideline on management of paraproteinemic demyelinating neuropathies. Report of a Joint Task Force of the European Federation of Neurological Societies and the Peripheral Nerve Society--first revision. J Peripher Nerv Syst 2010;15:185-95.

4. Beadon K, Guimarães-Costa R, Léger JM. Multifocal motor neuropathy. Curr Opin Neurol 2018;31:559-64.

5. Saperstein DS, Amato AA, Wolfe GI, et al. Multifocal acquired demyelinating sensory and motor neuropathy: the Lewis-Sumner syndrome. Muscle Nerve 1999;22:560-6. 